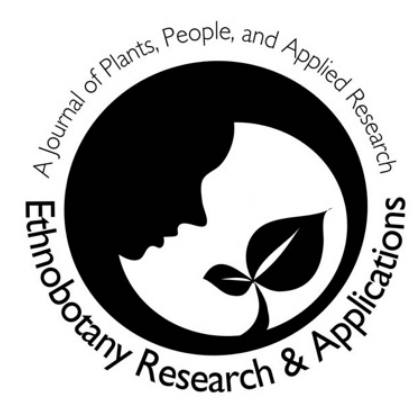

\title{
Alleviation of poverty through sustainable management and market promotion of medicinal and aromatic plants in Swat, Pakistan
}

Hazrat Sher, Ahmad Ali, Zahid Ullah and Hassan Sher

Correspondence

Hazrat Sher ${ }^{1}$, Ahmad Ali ${ }^{2}$, Zahid Ullah² and Hassan Sher ${ }^{2 *}$

${ }^{1}$ Department of Botany, GPG Jahanzeb College Swat, Khyber Pakhtunkhwa, Pakistan.

${ }^{2}$ Centre for Plant Sciences and Biodiversity, University of Swat, Khyber Pakhtunkhwa, Pakistan.

${ }^{*}$ Corresponding Author: hassan.botany@gmail.com

Ethnobotany Research and Applications 23:16 (2022)

\section{Research}

\begin{abstract}
Background: District Swat, Pakistan is a potential hub of medicinal and aromatic plants (MAPs) which can substantially contribute to social, economic and cultural uplift of local communities. Therefore, the present study was conducted to explore the market potential for MAPs in the study area.
\end{abstract}

Methods. Interviews were conducted with medicinal plant collectors, dealers and hakims to gather information about medicinal properties, pattern of collection and market status of MAPs in the study area. For this purpose, extensive field surveys were carried out in the study area and questionnaires were developed as a tool for interviews and data collection.

Results. The study reported 58, 38 and 64 plant species at collectors', dealers' and hakims' level. These plants are being used as medicines by local inhabitants to overcome their health problems and traded to subsidize low income for their basic needs. However, it was revealed that collectors had very little marketing skills and were unaware of high market value of their products, therefore, received minimum financial reward in the trade chain of medicinal plants. Increasing pressure on natural resources and decreasing trend in availability of important MAPs was also observed.

Conclusion: An urgent need to investigate links between overexploitation of species and quality of herbal medicines in the global market and to develop conservation strategies for MAPs is suggested. It is also recommended to develop training programs for collectors of medicinal plants in order to improve sustainability, trade monitoring and community participation in natural resource management.

Keywords. Medicinal plants, herbal market, quality trade monitoring, resource management

\section{Background}

Medicinal and aromatic plants (MAPs) not only contribute substantially to healthcare system but also serve as significant factor in livelihood security in rural mountainous settings (Astutik et al. 2019). MAPs are considered the most valuable among the vast array of non-timber forest products (Larsen et al. 2005). Traditionally used, plant derived medicine has been estimated worth US $\$ 83$ billion in the year 2008 (Shakya 2016), and in China alone the 
contribution from medicinal plants export is US $\$ 23.71$ billion (Ji et al. 2020; Uzun \& Koca 2020). Total income of poor households from the sale of wild medicinal herbs is between 15\%-30\% (Hamilton 2004). It has been estimated that at least $25 \%$ drugs in modern pharmacopeia and $18 \%$ drugs among the 150 highly prescribed medicine are plant-derived. About $50 \%$ of the global medicinal plants export is fulfilled by the Asian region alone, with China and India by for the largest export players in the market. Collection of medicinal plants from the wild or cultivation is an important livelihood source for many communities in several Asian countries including China, India, Pakistan, Nepal, Bangladesh, Myanmar, Iran, Turkey and Indonesia. In northwestern parts of Pakistan, particularly in Swat valley medicinal plants are the valuable natural economic resources and their export development through application of scientific knowledge may help in achieving the vision of economic growth (Nwafor et al. 2021). In the global MAPs market, Pakistan is amongst important countries which contribute to export of medicinal herbs to international medicinal plants markets (Hussain et al. 2009, Amjad et al. 2020, El-Assri et al. 2021). Mounting poverty can fuel commercial harvesting because more people driven by circumstances resort to herbal treatments and collecting medicinal plants to sell as a source of income (Sher et al. 2010).

Hakims (the traditional practitioners of Unani system of medicine) utilize many MAPs in their traditional formulations. They mostly obtain medicinal plants from the local collectors, sometimes collect themselves from the wild and often also obtain it from medicinal herbs dealers (pansars). At least 500 shops of medicinal plants dealers are present in various areas of district Swat. The collection and trade of MAPs species in the region are receiving increasing attention at both national and international levels (Ahmad et al. 2014). More than 350 medicinal plant species have been reported from district Swat, inhabiting a variety of habitat types. The contribution of Swat in Pakistan's MAPs market is not less than 25\% (Sher et al. 2017). However medicinal plant wealth and its future production, potential for commercialization and possible contribution to national economy is unclear. Due to shortcomings in present system of MAPs collection and trading, unsustainable harvesting of several species has rendered them threatened with severe population decline in the study area. Increasing demand for a wide range of MAPs have resulted in at least 10,000 MAPs becoming currently threatened on the global scale (Hamilton 2004). Another important aspect of medicinal plants is their quality, to which no attention has been paid and presently the collectors harvest medicinal plants unsustainably and improperly, and often do not know that how and which part of a plant needs to be collected. Additionally, there is lack of knowledge regarding time of collection and no attention is paid to the effect of storage on efficacy. The lack of knowledge concerning economic value of MAPs has led to their mismanagement and least profitable exploitation by local residents, as well as visiting collectors (Van Wyk \& Prinsloo 2018).

Therefore, we engaged in the aim to examine the status of MAPs marketing alongside linkages in the trade chain from collectors to consumers. Secondly, we aimed to analyze the market trends for medicinal herbs in Swat of the last decade, and to identify potential problems associated with current decline in the market share of MAPs in the area.

\section{Materials and Methods}

\section{The study area}

The study area district Swat is located in the extreme North-western parts of Pakistan and lies between $35.2227^{\circ}$ $\mathrm{N}, 72.4258^{\circ} \mathrm{E}$. The area is mountainous and having an area of $5337 \mathrm{~km} 2$, with a total population of about 2.3 million, and an elevation gradient that varies from 600-6069 m (GoP, 2017). The highest peak (Falakser, $6069 \mathrm{~m}$ ) occupies the floristically rich southern extinction of Hindu raj of the Hindu Kush series. The population of the valley is mostly dependent upon agriculture, tourism, government, and private jobs, as well as livestock production in upper Swat. The valleys in upper Swat include forests and pastures occupied by hundreds of households of nomadic herdsmen who come to these valleys in May and June each year, for grazing their livestock during summer, and migrate to the lower districts in October and November. Humid summers and chilly winters make the valley conducive for the growth and development of natural resources including medicinal herbs (Khan et al. 2018). A considerable part of the population is gathering different forest resources including MAP species in the valley, which play an important role in the supply and trade of medicinal plants in the country. However, information allowing a more sustainable utilization of MAPs for the economic development of local community is urgently needed.

Current study was therefore, conducted during 2017-2018 through field work after consulting topographical maps and other related information obtained from the Forest department, Swat, KP, Pakistan. In addition, technical data was obtained from various sources including MAPs dealers and brochures/pamphlets. The area was accordingly divided into various localities and then collectors of medicinal plants, dealers, and hakims, were interviewed after obtaining their free prior informed consent. Technical literature relating to collection, trading, processing, and 
national and international marketing was consulted in different areas including the main city Mingora, Swat. Information and data from various local elder and knowledgeable people in different places of the valley about medicinal plants and poverty situation were also obtained through formal interviews and personal frequent visits at regular intervals to different localities in the study area. Data was also collected from MAPs exporters, local herbal dealers, hakims (local healers using Unani system of medicine) using questionnaires and herbal pharmaceuticals companies.

\section{Interviews}

Extensive field surveys were carried out throughout the study area and semi-structured questionnaires were developed and used as a tool for interviews and data collection following methods described in Sher et al. (2017). Each questionnaire was divided into two parts, the first part was pertinent to ethnographic information, such as name, locality, age, education and profession of the informant. The second part was specific to the pattern of collection, marketing, and use of medicinal and aromatic plants. Generally, the respondents were elderly persons and their age group varied from 40 to 70 years. Women were also interviewed, however, due to cultural restrictions in the area, information about medicinal herbs from women could normally only be obtained in the presence of their family members. The collectors were asked how they collected plants, from where, and to whom they sold the plants. Respondents were also asked about their annual revenues earned from the sale of the plants and return of the work invested. Data on marketing of MAPs was gathered from local experienced persons by enlisting the marketable and best priced species known to them. Information on the market value of the plants was collected from local collectors and shopkeepers.

A specific questionnaire was also used for interviewing traders and hakims. Data/information was also obtained from about 200 MAPs collectors/farmers, 50 local dealers and exporters and 8 active herbal pharmaceuticals companies exporting medicinal plants in the country. Information was gathered about major items of sale, source and origin of crude herbs, approximate quantities of material transacted per year, annual income and common problems encountered during the process. Information was also gathered to establish most important widely used medicinal plants species, origin of crude herbs and their standard requirements from collectors/dealers regarding formulation/products.

\section{General poverty situation}

Direct estimation of poverty was determined following the method of Rasul et al. (2012), by carrying out interviews with local people (collectors), and it was generally observed that poverty existed. It was also found that large size families with few earning members was a symptom of poverty which has led to increasing pressure on natural resources including MAPs. Estimates suggests that 27.3\% people in district Swat are living below the poverty line, and MAPs are the source of their primary cash income as well as support their household livelihoods. At present, the poverty rate has increased due to multiple challenges including natural disasters like the impact of 2010 flood.

\section{Observations and documentation}

Effects of current harvesting practices on the status of each medicinally and commercially valuable plant species was also studied by comparing current data to 20 years old records with respect to population size and status. The effect of current harvest impact on the population size of MAPs was judged based on the changes in distance that local collectors covered for collection in past as compared to the present.

Guided by selected male members of the community, the team were shown local habitat and availability of medicinal herb through transect walk. This visual experience enabled the botanical/Latin identification of these herbs and recording of other indigenous species unknown to the community to have medicinal properties. Collectors with their local names identified all MAPs, and later voucher specimens were identified based on pertinent literature (Nasir and Ali 1971-89). The voucher specimens were deposited to Swat University Herbarium (SWAT).

\section{Results}

Our study reported 58, 38 and 64 plant species at collectors', dealers', and hakims' level. These plants were being used as medicines by local inhabitants to overcome their health problems and traded to subsidize low income for their basic needs.

\section{Collectors}


Collectors of MAPs species served as an important source of information which comprised of local men, women, children and visiting collectors, most of them illiterate. The survey also showed that local women and children mostly collected plants, with men and visiting collectors/outsiders in the minority (Figure 1). It was observed that collectors harvested medicinal plants unsustainably and improperly, to the extent that they did not know that how and which part of the plant needed to be collected. Additionally, there was a lack of knowledge about correct time of collection of MAPs species. Furthermore, lack of knowledge concerning economic value of MAPs led to their mismanagement and least profitable exploitation, not only by local residents but also visiting collectors.

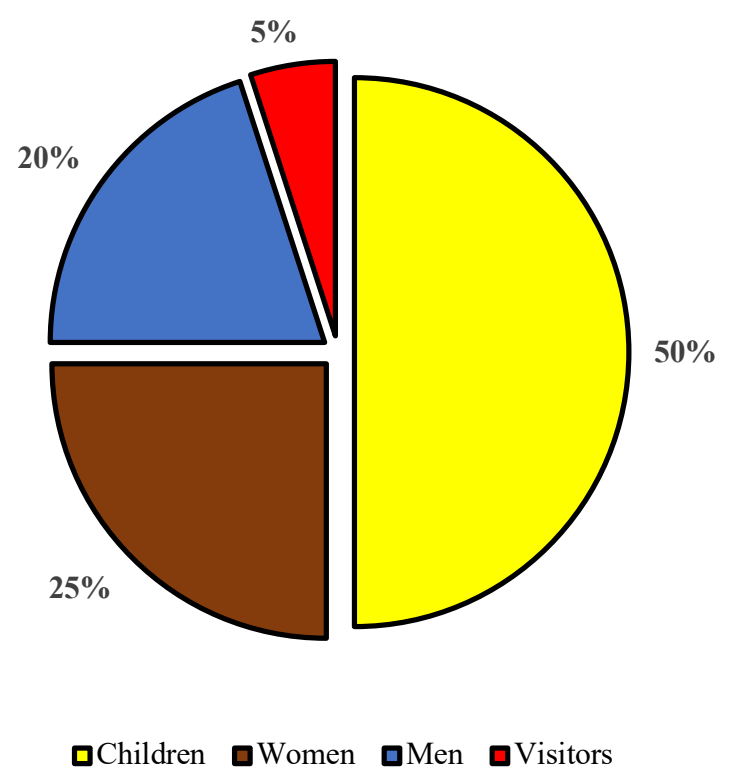

Figure 1. Percentage of different collectors involved in collection of medicinal herbs in district Swat.

\section{Linkages in market chain and general trade pattern of medicinal herbs in district Swat}

District Swat is the collection and trade center for many medicinal and economically valuable herbs and supply sizeable quantities of plant material to both national and international markets in the country and abroad. However, it was observed that trade and collection was not systematic and varied from species to species. The dealers from larger cities including Karachi, Lahore, Islamabad, and Peshawar, send representatives and put in a demand for certain species of the area. Local dealers pass the message to their agents who disperse in the hillside areas and inform shopkeepers and collectors about demand for those particular species. As a result, some profit accrues to each of these 'middlemen' and the hillside community who collect large quantities of medicinal plants receive very little remuneration or reward for the collection.

Analysis of the exact volume and value of annual trade about medicinal plants is suggested to be tentative due to changeability, hesitation, and uncertainty of people to show the real price and to tell truth about their income. Materials was generally traded in dry or semi-dried form, and rarely in fresh form, but often not properly packed. The survey also revealed that Swat was not only export center for economically and medicinally important plant species, but medicinal herbs are also imported from the major cities of Pakistan including Peshawar, Lahore, Quetta and Gilgit, and from abroad. Figure 2 shows market routes (channels) through which MAPs are exported from various localities in the study area (Swat) from collection sites and other areas including neighbor districts bordered by investigated area to local, national, and international markets of medicinal plants.

\section{Collection and trade of marketable medicinal plants in district Swat}

Three hundred collectors in different valleys of district Swat were interviewed about collection and trade of medicinal and aromatic plants. Collectors were found gathering 58 different MAPs species during various times of the year (Table 1). Study revealed that most of the plants (40 spp.) were gathered in summer, followed by spring (27 spp.), and fewer species (7 spp.) were collected throughout the year, mainly from high-elevation areas. Government has neither proper control nor a permit system for collection of MAPs and both traditional and visiting collectors were gathering medicinal herbs. 


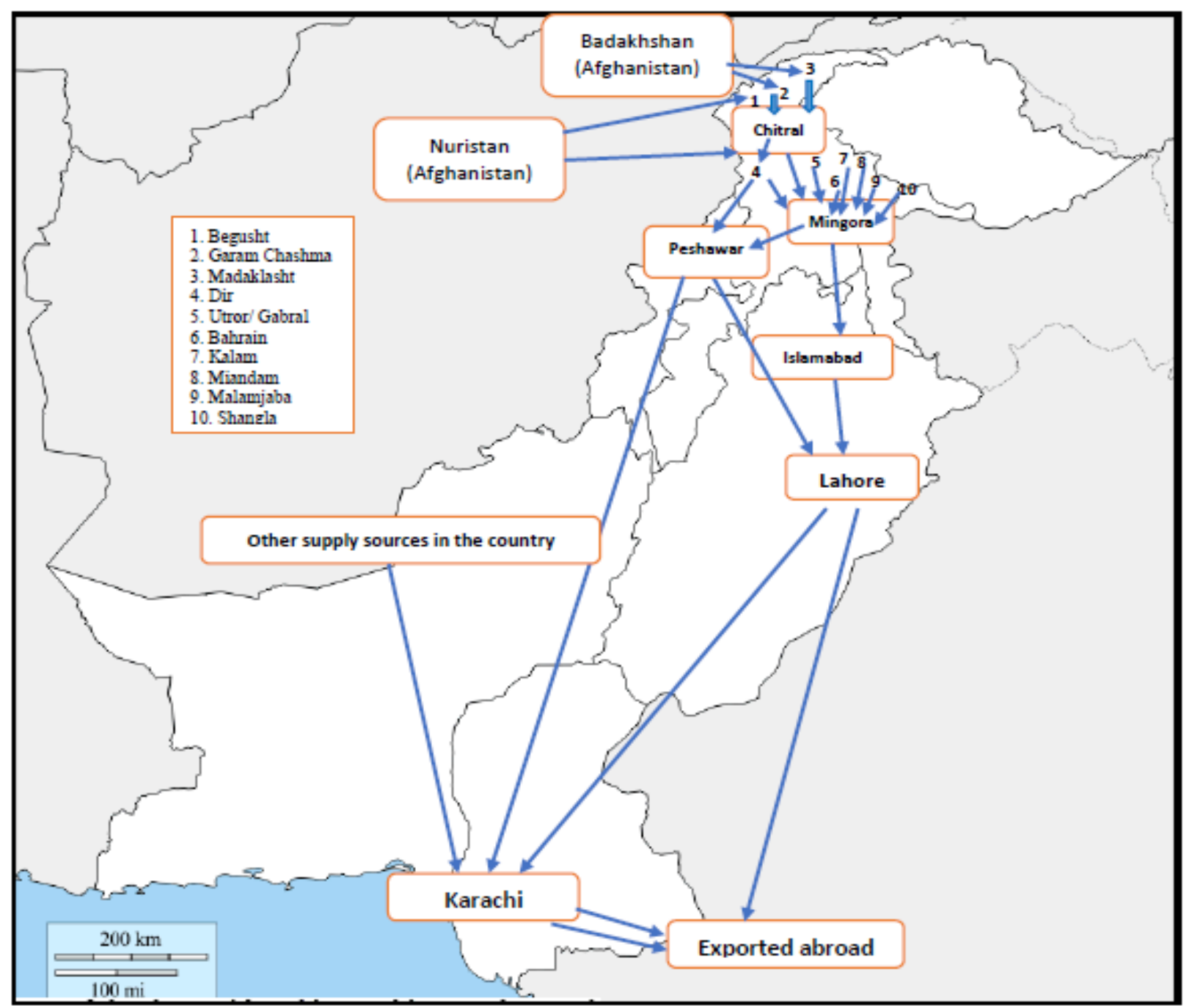

Figure 2. Market channels from collection sites and other areas to local, national and international markets.

\section{Trade of medicinal plants at dealers' level in district Swat}

Approximately 38 species of medicinal and aromatic herbs included in this study were widely traded by dealers in the study area (Table 2). Majority of medicinal plants were of local origin; however, a number of medicinal plants was imported from other districts of Malakand Division such as Shangla, Dir and Buner to the main city of Swat (Mingora) to reach to the national markets, e.g., Peshawar, Islamabad, Lahore and Karachi (Figure 2). The study reported that price of each material increased in the market chain from collector's level to national and international markets.

\section{Trade of medicinal plants at Hakim's level in district Swat}

It was observed that hakims (traditional healers) were collecting and trading a greater number of medicinal herbs than dealers of the area. The trade of medicinal plants at hakim's level was much more complex and heterogeneous than at dealers' level. The study reported that Hakims bought only a few species of indigenous medicinal plants from local markets. They mostly collect these plants themselves or employed their patients or children. Hakims generally did not give any remuneration to the collectors, but as a reward they treated the patients free of charge or just gave a few tonic tablets to them. They used approximately $20 \%$ species of indigenous medicinal plants in their clinics and shops at domestic level, while approximately $80 \%$ plant materials were exported to big herbal pharmaceuticals companies and dealers of Lahore, Karachi, Islamabad, and Peshawar. In this way, hakims made a good profit from the sale of medicinal plants (Table 3 and Figure 3). 
Table 1. List of medicinal and aromatic plants (MAPs) traded in herbal markets of district Swat

\begin{tabular}{|c|c|c|c|c|c|c|c|c|c|}
\hline Botanical Name & Family & Local Name & Part used & Form & $\begin{array}{l}\text { Collected } \\
\text { since(year) }\end{array}$ & $\begin{array}{l}\text { Annual } \\
\text { collection } \\
\text { in mound }\end{array}$ & $\begin{array}{l}\text { Collection } \\
\text { Time }\end{array}$ & $\begin{array}{l}\text { Price } \\
\text { (Rs/Kg) }\end{array}$ & $\begin{array}{l}\text { Voucher } \\
\text { No. }\end{array}$ \\
\hline Adiantum capillusveneris L. & Pteridaceae & Persushan & WP & Dried & $25-35$ & $650-700$ & Apr-Sep. & 20 & HS-01 \\
\hline Aconitum heterophyllum Wall. & Ranunculaceae & Zaharmora & $\mathrm{R}$ & Fresh & $25-30$ & 60 & May-Sep & 75 & HS-02 \\
\hline Aconitum laeve Royle. & Ranunculaceae & Zaharmora & $\mathrm{R}$ & Fresh & $25-30$ & 60 & May-Sep & 75 & $\mathrm{HS}-03$ \\
\hline Ajuga integrifolia Buch.-Ham. & Lamiaceae & Booti/Gooti & $\mathrm{S}, \mathrm{L}$ & Dried & 15 & $100-140$ & May-Oct. & 20 & HS-04 \\
\hline Achillea millefolium $\mathrm{L}$. & Asteraceae & Barajassif & WP & Fresh & 10 & $6-8$ & May-Sep. & 20 & HS-05 \\
\hline Allium sativum $\mathrm{L}$. & Amaryllidaceae & Ouga & $\mathrm{B}$ & Fresh & 30 & $15-25$ & May-Sep. & 100 & HS-06 \\
\hline Arisaema flavum (Forsk) Schott. & Araceae & Marjarrai & $\mathrm{C}$ & Fresh & 15 & $3-5$ & May-Sep. & 70 & HS-07 \\
\hline Acorus calamus L. & Acoraceae & Skhawaja & $\mathrm{Rh}$ & Fresh & $25-30$ & $150-180$ & Jan- Dec. & 65 & $\mathrm{HS}-08$ \\
\hline Artemisia vulgaris L. & Asteraceae & Tarkha & $\mathrm{Fl}, \mathrm{YS}, \mathrm{L}$ & Fresh & 15 & $50-100$ & Apr-Jul & 35 & HS-09 \\
\hline Berberis lycium Royle. & Berberidaceae & Toor kwaray & $\mathrm{Ba}, \mathrm{S}, \mathrm{R}$ & Fresh & $20-30$ & $200-260$ & Jan- Dec. & 60 & $\mathrm{HS}-10$ \\
\hline Bergenia ciliata (Haw.) Sternb. & Saxifragaceae & $\begin{array}{l}\text { Ghatpana/ Zakhme- } \\
\text { hayat }\end{array}$ & $\mathrm{Rh}$ & Dried & $20-30$ & $250-280$ & May-Sep. & 45 & HS-11 \\
\hline $\begin{array}{l}\text { Persicaria amplexicaulis (D. Don) } \\
\text { Ronse Decr. }\end{array}$ & Polygonaceae & Tarwa Panra & $\mathrm{Rh}$ & Dried & $20-30$ & $1500-1800$ & May-Sep. & 120 & HS-12 \\
\hline Bunium persicum B.Fedstch. & Apiaceae & Zeera & $\mathrm{Fr}$ & Dried & 25 & $30-50$ & Apr-Jun. & 250 & HS-13 \\
\hline Caltha alba Jacquem. & Ranunculaceae & Makhan Path & $\mathrm{Fl}$ & Fresh & 20 & $10-15$ & Apr-May & 30 & HS-14 \\
\hline Cichorium intybus L. & Asteraceae & Han/ Kasni & $R, L$ & Fresh & 20 & $550-600$ & Feb-May & 25 & HS-15 \\
\hline Colchicum luteum Baker. & Colchicaceae & Suranjan talakh & $\mathrm{B}$ & Fresh & 30 & $80-100$ & Mar-May & 300 & HS-16 \\
\hline Calotropis procera (Wild.) R. Brown. & Apocynaceae & Ak/Spalmai & $\mathrm{R}, \mathrm{Fl}, \mathrm{L}$ & Fresh & 15 & $120-16$ & Apr-Jul. & 45 & HS-17 \\
\hline Cuminum cyminum $\mathrm{L}$. & Apiaceae & Spina zeera & $\mathrm{Fr}$ & Dried & 25 & $5-8$ & Apr-Jun & 200 & HS-18 \\
\hline Caralluma tuberculata N.E.Br. & Apocynaceae & Pamankay & WP & Fresh & 25 & $320-360$ & Feb-Apr. & 40 & HS-19 \\
\hline Cedrus deodara Roxb ex Lambert. & Pinaceae & Diyar/Ranzra & $\mathrm{Gu}$ & Dried & 20 & $80-100$ & Apr-Nov. & 150 & $\mathrm{HS}-20$ \\
\hline Corydalis govaniana Wall. & Papaveraceae & Mamera & $\mathrm{Fl}, \mathrm{R}$ & Fresh & 20 & $350-380$ & Apr-Sep & 50 & HS-21 \\
\hline Carum copticum $\mathrm{L}$. & Apiaceae & Ajwain & $\mathrm{Fr}$ & Dried & 20 & $400-450$ & Apr-Jun & 55 & HS-22 \\
\hline Diospyros lotus L. & Ebenaceae & Toor amlok & $\mathrm{Fr}$ & Dried & 30 & 75,000 & Sep-Nov & 20 & HS-23 \\
\hline Dryopteris juxtaposita Christ. & Dryopteris & Kwanjay & $\mathrm{YP}$ & Fresh & 30 & $400-460$ & Apr-Aug & 25 & HS-24 \\
\hline Dioscorea deltoidea Wall. ex Griseb. & Dioscoreaceae & Kanis & $\mathrm{Rh}$ & Fresh & 30 & $80-100$ & Apr-Sep & 45 & HS-25 \\
\hline Ficus carica $\mathrm{L}$. & Moraceae & Inzar & $\mathrm{Fr}$ & Fresh & 20 & $200-220$ & May-Sep & 35 & HS-26 \\
\hline
\end{tabular}


Ethnobotany Research and Applications

\begin{tabular}{|c|c|c|c|c|c|c|c|c|c|}
\hline Fagonia cretica L. & Zygophyllaceae & Azghakay & S & Fresh & 25 & $100-120$ & May-Oct & 65 & HS-27 \\
\hline Fumaria indica (Hausskn.) Pugsley & Papveraceae & Papra & $\mathrm{S}, \mathrm{L}$ & Dried & 15 & $150-180$ & Apr-Jun & 25 & HS-28 \\
\hline Geranium wallichianum D.Don. & Geraniaceae & Sragarrai & $\mathrm{R}$ & Fresh & 20 & $50-80$ & May-Sep & 50 & HS-29 \\
\hline Hedera helix $\mathrm{L}$. & Aralliaceae & Palul zelai & $\mathrm{L}$ & Fresh & 15 & 100 & Jan-Dec & 90 & $\mathrm{HS}-30$ \\
\hline Juglans regia $\mathrm{L}$. & Juglandaceae & Ghoz & $\mathrm{Fr}$ & Dried & 30 & 90,000 & Sep-Oct & 150 & HS-31 \\
\hline Justicia adhatoda L. & Acanthaceae & Bekan & L & Fresh & 20 & $380-400$ & Apr-Nov & 40 & HS-32 \\
\hline Morchella esculenta L. & Morchellaceae & Guji/Khosai & WP & Fresh/ & 30 & 1000 & Mar-May & 10,000 & HS-33 \\
\hline Mentha longifolia (L.) L. & Lamiaceae & Velanay & YS & Fresh & 20 & 350 & Apr-Aug & 45 & HS-34 \\
\hline Myrtus communis L. & Myrtaceae & Manro & L & Fresh & 20 & 550 & Feb-May & 25 & $\mathrm{HS}-35$ \\
\hline Malva neglecta Wall. & Malvaceae & Panerak & YS & Fresh & 15 & 450 & Apr-Aug & 25 & HS-36 \\
\hline Morus alba L. & Moraceae & Spin tout & $\mathrm{Fr}$ & Fresh & 20 & 250 & May-Sep & 45 & HS-37 \\
\hline Morus nigra L. & Moraceae & Tour tout & $\mathrm{Fr}$ & Fresh & 20 & 250 & May-Sep & 45 & HS-38 \\
\hline Onosma echioides Wall. & Boraginceae & Ratanjut & WP & Fresh & 20 & $6-8$ & May-Sep & 55 & HS-39 \\
\hline Paeonia emodi Wall. & Paeoniaceae & Mamekh & $\mathrm{Rh}$ & Dried & 30 & $350-380$ & Apr-Sep & 60 & $\mathrm{HS}-40$ \\
\hline $\begin{array}{l}\text { Sinopodophyllum hexandrum (Royle) } \\
\text { T.S. Ying }\end{array}$ & Berberidaceae & Bankakrri/ Kakora & $\mathrm{Rh}$ & Dried & 30 & $2500-280$ & Apr-Sep & 180 & HS-41 \\
\hline Pistacia integerrima St. ex Brand. & Anacardiaceae & Shnai & $P$ & Fresh & 30 & $250-280$ & May-Sep & 200 & HS-42 \\
\hline Polygonatum multiflorum (L.) All. & Asparagaceae & Nor-e-alam & $\mathrm{Rh}$ & Fresh & 30 & $180-220$ & Apr-Sep & 75 & HS-43 \\
\hline Polygonatum verticillatum (L.) All. & Asparagaceae & Nor-e-alam & $\mathrm{Rh}$ & Fresh & 30 & $250-300$ & Apr-Sep & 75 & HS-44 \\
\hline Pinus roxburghii Sargent. & Pinaceae & Nakhtar & $\mathrm{Gu}$ & Dried & 25 & $100-150$ & Jan-Dec & 160 & HS-45 \\
\hline Rheum emodi Wall. & Polgonaceae & Rewand chini & $\mathrm{R}$ & Fresh & 25 & $80-120$ & Apr-Oct & 40 & HS-46 \\
\hline Rosa moschata Benth. & Rosaceae & Gulab & $\mathrm{Fl}$ & Dried & 20 & $150-200$ & May-Aug & 45 & HS-47 \\
\hline Rheum webbianum Royle. & Polgonaceae & Chotial & Rh, L & Fresh & 30 & $80-100$ & May-Sep & 50 & HS-48 \\
\hline Thymus linearis Benth. & Lamiaceae & Ghra sperkai & WP & Dried & 20 & $40-60$ & May-Aug & 40 & HS-49 \\
\hline Taxus wallichaina Zucc. & Taxaceae & Banya & L & Fresh & 15 & 500 & Apr-Oct & 60 & HS-50 \\
\hline Skimmia laureola Sieb \& Zuck. & Rutaceae & Nazar panra & $\mathrm{L}$ & Fresh & 30 & 1000 & Apr-Sep & 35 & HS-52 \\
\hline Jurinea dolomiaea Boiss. & Asteraceae & Sharrshami & $\mathrm{Rh}$ & Fresh & 30 & 80 & May-Sep & 35 & HS-53 \\
\hline Viola canescens Wall. & Violaceae & Banafsha & $\mathrm{L}, \mathrm{Fl}$ & Dried & 30 & $100-1200$ & Mar-May & 600 & HS-53 \\
\hline Valeriana jatamansi Jones. & Caprifoliaceae & Mushkebala & $\mathrm{Rh}$ & Fresh & 30 & $1500-1800$ & Apr-Sep & 200 & HS-54 \\
\hline Ziziphus jujuba Mill. & Rhamnaceae & Markhanai & $\mathrm{Fr}$ & Dried & 20 & $25,000-$ & Jul-Sep & 35 & HS-55 \\
\hline Zanthoxylum armatum DC. & Rutaceae & Dambara & $\mathrm{Fr}$ & Fresh & 20 & $120-160$ & May-Aug & 25 & HS-56 \\
\hline
\end{tabular}

Where: Ba, bark; B, Bulb; C, corm; Fr, fruit; $\mathbf{F l}$, flower; Gu, gum; L, leaves; P, pod; R, root; Rh, rhizome; S, stem; WP, whole plant; YP, young plant; YS, young shoot. 
Table 2. Marketable MAPs price per $\mathrm{kg}$ traded by major dealers of district Swat

\begin{tabular}{|c|c|c|c|c|c|c|c|c|c|}
\hline Botanical Name & $\begin{array}{l}\text { Traded } \\
\text { part }\end{array}$ & Form & $\begin{array}{l}\text { Traded } \\
\text { Since } \\
\text { (year) }\end{array}$ & $\begin{array}{l}\text { Annual } \\
\text { trade } \\
\text { (amount) }\end{array}$ & $\begin{array}{l}\text { Purchase Price } \\
\text { (Rs/Kg) }\end{array}$ & $\begin{array}{l}\text { Retail Price } \\
\text { (Rs/Kg) }\end{array}$ & $\begin{array}{l}\text { Wholesale price } \\
\text { (Rs/Kg) }\end{array}$ & $\begin{array}{l}\text { National } \\
\text { market } \\
\text { Price/kg }\end{array}$ & $\begin{array}{l}\text { International } \\
\text { Market Price } \\
\text { (USD/kg) }\end{array}$ \\
\hline Adiantum capillusveneris $\mathrm{L}$. & WP & Dried & 40 & 850 & 20 & 40 & 55 & 65 & - \\
\hline Aconitum heterophyllum Wall. & $\mathrm{R}$ & Dried & 35 & 120 & 75 & 160 & 280 & 350 & 40 \\
\hline Aconitum laeve Royle. & $\mathrm{R}$ & Dried & 35 & 120 & 75 & 160 & 280 & 350 & 40 \\
\hline Allium sativum $\mathrm{L}$. & B & Dried & 40 & 21 & 100 & 150 & 200 & 250 & - \\
\hline Asparagus adscendens Roxb. & $\mathrm{Rh}$ & Dried & 30 & 25 & 1300 & 1600 & 2000 & 2500 & 35 \\
\hline Acorus calamus L. & $\mathrm{Rh}$ & Dried & 30 & 450 & 65 & 140 & 300 & 450 & - \\
\hline Artemisia maritima & $\mathrm{Fl}, \mathrm{YS}, \mathrm{L}$ & Dried & 25 & 120 & 35 & 55 & 70 & 120 & - \\
\hline Berberis lyceum Royle & $\mathrm{Ba}, \mathrm{S}, \mathrm{R}$ & Dried & 40 & 400 & 60 & 80 & 110 & 150 & - \\
\hline Bergenia ciliata (Haw.) Sternb. & $\mathrm{Rh}$ & Dried & 40 & 400 & 45 & 65 & 120 & - & - \\
\hline $\begin{array}{l}\text { Persicaria amplexicaulis (D. } \\
\text { Don) Ronse Decr. }\end{array}$ & $\mathrm{Rh}$ & Dried & 30 & 2000 & 120 & 150 & 200 & 250 & 18 \\
\hline Bunium persicum B.Fedstch. & $\mathrm{Fr}$ & Dried & 15 & 70 & 250 & 280 & 300 & 450 & - \\
\hline Colchicum luteum Baker. & $\mathrm{B}$ & Dried & 40 & 50 & 300 & 400 & 480 & 650 & - \\
\hline $\begin{array}{l}\text { Cedrus deodara Roxb ex } \\
\text { Lambert. }\end{array}$ & $\mathrm{Gu}$ & Dried & 15 & 50 & 150 & 180 & 200 & 250 & - \\
\hline Corydallis govaniana Wall. & $\mathrm{Fl}, \mathrm{R}$ & Dried & 35 & 16 & 50 & 60 & 80 & 120 & - \\
\hline Diospyros lotus L. & $\mathrm{Fr}$ & Dried & 35 & 80,000 & 20 & 35 & 65 & - & - \\
\hline Dryopteris juxtapostia Christ. & $\mathrm{YP}$ & Dried & 10 & 400 & 25 & 40 & 45 & - & - \\
\hline $\begin{array}{l}\text { Dioscorea deltoidea Wall. ex } \\
\text { Griseb. }\end{array}$ & $\mathrm{Rh}$ & Dried & 40 & 280 & 45 & 80 & 150 & 250 & 32 \\
\hline Geramium wallichianum L. & $\mathrm{R}$ & Dried & 35 & 17 & 50 & 65 & 90 & - & - \\
\hline Juglans regia L. & $\mathrm{Fr}$ & Dried & 40 & 75000 & 150 & 180 & 200 & 250 & 40 \\
\hline Morchella esculenta L. & WP & Dried & 35 & 350 & 10,000 & 16,000 & 25,000 & 30,000 & 170 \\
\hline Mentha longilfolia (L.) L. & YS & Dried & 20 & 350 & 45 & 65 & 80 & - & - \\
\hline Paeonia emodi Wall. & $\mathrm{Rh}$ & Dried & 40 & 500 & 60 & 75 & 125 & 180 & 30 \\
\hline $\begin{array}{l}\text { Sinopodophyllum hexandrum } \\
\text { (Royle) T.S. Ying }\end{array}$ & $\mathrm{Rh}$ & Dried & 40 & 350 & 180 & 300 & 400 & 500 & 50 \\
\hline $\begin{array}{l}\text { Pistacia integerrima St. ex } \\
\text { Brand. }\end{array}$ & Pod & Dried & 30 & 500 & 200 & 380 & 450 & 700 & - \\
\hline $\begin{array}{l}\text { Polygonatum multiflorum (L.) } \\
\text { All. }\end{array}$ & $\mathrm{Rh}$ & Dried & 35 & 450 & 75 & 150 & 250 & - & - \\
\hline
\end{tabular}


Ethnobotany Research and Applications

\begin{tabular}{|c|c|c|c|c|c|c|c|c|c|}
\hline $\begin{array}{l}\text { Polygonatum verticillatum (L.) } \\
\text { All. }\end{array}$ & $\mathrm{Rh}$ & Dried & 35 & 450 & 75 & 160 & 280 & - & - \\
\hline Rheum emodi Wall. & $\mathrm{R}$ & Dried & 25 & 400 & 40 & 55 & 70 & - & - \\
\hline Rosa moschata Benth. & $\mathrm{Fl}$ & Dried & 30 & 250 & 45 & 60 & 75 & - & - \\
\hline Rheum webbianum Royle & Rh, L & Dried & 25 & 450 & 50 & 65 & 80 & - & - \\
\hline Taxus wallichiana Zucc. & $\mathrm{L}$ & Dried & 20 & 700 & 60 & 100 & 180 & 300 & - \\
\hline Skimmia laureola Sieb \& Zuck. & $\mathrm{L}$ & Dried & 10 & 800 & 35 & 50 & 65 & - & - \\
\hline Jurinea dolomiaea Boiss. & $\mathrm{Rh}$ & Dried & 30 & 60 & 35 & 50 & 65 & - & - \\
\hline Viola canescens Wall. & $\mathrm{L}, \mathrm{Fl}$ & Dried & 40 & 500 & 600 & 750 & 800 & 1200 & 14 \\
\hline Valeriana jatamansi Jones & $\mathrm{Rh}$ & Dried & 40 & 2000 & 200 & 280 & 400 & 600 & 7 \\
\hline Ziziphus jujuba L. & $\mathrm{Fr}$ & Dried & 25 & 45000 & 35 & 65 & 90 & - & - \\
\hline Zanthoxyllum armatum DC. & $\mathrm{Fr}$ & Dried & 20 & 200 & 30 & 40 & 45 & - & - \\
\hline
\end{tabular}

Where: Ba, bark; C, corm; Fr, fruit; Fl, flower; Gu, gum; L, leaves; $\mathbf{P}$, pod; R, root; $\mathbf{R h}$, rhizome; S, stem; WP, whole plant; YP, young plant; YS, young shoot. 
Table 3. Marketable species of medicinal plants used/traded by hakims and their contribution to poverty reduction in district Swat

\begin{tabular}{|c|c|c|c|c|c|c|}
\hline Botanical Name & Local Name & Habit & Market demand & Part used & Price Rs/kg & Treatment of diseases \\
\hline Adiantum capillusveneris $\mathrm{L}$. & Persushan & Herb & Persistent & WP & 80 & Cooling agent and febrifuge. \\
\hline Achyranthes aspera L. & Gishkay & Herb & Persistent & $W p$ & 70 & Rheumatism \\
\hline Aconitum heterophyllum Wall. & Zaharmora & Herb & Increased & $\mathrm{R}$ & 600 & $\begin{array}{l}\text { Astringent, stomachic and } \\
\text { aphrodisiac. }\end{array}$ \\
\hline Aconitum laeve Royle. & Zaharmora & Herb & Increased & $\mathrm{R}$ & 800 & $\begin{array}{l}\text { Astringent, stomachic and } \\
\text { aphrodisiac }\end{array}$ \\
\hline Ajuga integrifolia Buch.-Ham. & Booti/Gooti & Herb & Increased & WP & 90 & Fever \\
\hline Ajuga parviflora Benth. & Booti & Herb & Persistent & WP & 90 & Fever, diuretic \\
\hline Asparagus adscendens Roxb. & Muslisufaid & Shrub & Increased & $\mathrm{Rh}$ & 3500 & Aphrodisiac/diuretic \\
\hline Asparagus officinalis $\mathrm{L}$. & Muslisufaid & Herb & Increased & $\mathrm{S}$ & 250 & Aphrodisiac/diuretic \\
\hline Acorus calamus L. & Skhawaja & Herb & Increased & $\mathrm{Rh}$ & 120 & Stomachic and tonic flatulent \\
\hline Arisaema flavum (Forsk) Schott. & Marjarrai & Herb & Increased & $\mathrm{R}$ & 450 & Rheumatism \\
\hline $\begin{array}{l}\text { Seriphidium brevifolium (Wall. } \\
\text { ex DC.) Ling \& Y.R. Ling }\end{array}$ & Tarkha & Herb & Persistent & $\mathrm{R}, \mathrm{L}$ & 220 & Stomachic \\
\hline Artemisia maritima $\mathrm{L}$. & Tarkha & Herb & Persistent & $\mathrm{Fl}, \mathrm{YS}, \mathrm{L}$ & 110 & Deworming/fever \\
\hline Artemisia vulgaris $\mathrm{L}$. & Tarkha & Herb & Increased & $\mathrm{R}, \mathrm{L}, \mathrm{Fl}$ & 120 & Fever \\
\hline Berberis lycium Royle & Spin Kwaray & Shrub & Increased & $\mathrm{Ba}, \mathrm{S}, \mathrm{R}$ & 180 & $\begin{array}{l}\text { Jaundice and wound healing } \\
\text { antibiotic }\end{array}$ \\
\hline Bergenia ciliata (Haw.) Sternb. & Ghatpana/Bogandi & Herb & Persistent & $\mathrm{Rh}$ & 90 & Goit pain/tonic \\
\hline Bunium persicum B.Fedstch. & Zeera & Herb & Increased & $\mathrm{Fr}$ & 500 & Carminatives and stimulant \\
\hline Cuscuta reflexa Roxb. & Zelai & Parasite/climber & - & WP & 65 & Diabetic, fever and carminatives \\
\hline Cichorium intybus L. & Han/Kasni & Herb & Persistent & $\mathrm{R}, \mathrm{L}$ & 55 & Jaundice and Fever \\
\hline Colchicum luteum Baker. & Suranjan-e-talkh & Herb & Increased & C & 550 & $\begin{array}{l}\text { Laxative, aphrodisiac and } \\
\text { rheumatism }\end{array}$ \\
\hline Calotropis procera & Ak/Spalmai & Shrub & Persistent & $\mathrm{R}, \mathrm{Fl}, \mathrm{L}$ & 200 & Dysentery and toothache \\
\hline Cuminum cyminum & Spina zeera & Herb & Persistent & $\mathrm{Fr}$ & 200 & Carminatives and stimulant \\
\hline Caralluma edulis & Pamankay & Herb & Increase & WP & 120 & Diabetic \\
\hline $\begin{array}{l}\text { Cedrus deodara Roxb ex } \\
\text { Lambert. }\end{array}$ & Diyar/Ranzra & Tree & Increased & $\mathrm{Gu}$ & 2000 & Diuretic and fever \\
\hline Corydallis govaniana Wall. & Mamera & Herb & Persistent & $\mathrm{Fl}, \mathrm{R}$ & 200 & Eye diseases \\
\hline Carum copticum & Ajwain & Herb & Persistent & $\mathrm{Fr}$ & 120 & $\begin{array}{l}\text { Carminatives, astringent and } \\
\text { stimulant }\end{array}$ \\
\hline
\end{tabular}


Ethnobotany Research and Applications

\begin{tabular}{|c|c|c|c|c|c|c|}
\hline Diospyros lotus L. & Toor amlok & Tree & Increased & $\mathrm{Fr}$ & 50 & Dysentery and astringent \\
\hline $\begin{array}{l}\text { Dioscorea deltoidea Wall. ex } \\
\text { Griseb. }\end{array}$ & Kanis & Climber & Decreased & $\mathrm{Rh}$ & 90 & Bronchitis \\
\hline $\begin{array}{l}\text { Ephedra gerardiana Wall. es } \\
\text { Stapf }\end{array}$ & Someni & Shrub & Increased & $\mathrm{Ba}, \mathrm{Fr}, \mathrm{L}, \mathrm{R}$ & 300 & Asthma \\
\hline Ficus carica L. & Inzar & Tree & Persistent & $\mathrm{Fr}$ & 160 & Kidney stone \\
\hline Fagonia cretica L. & Azghakay & Herb & Persistent & $\mathrm{S}$ & 120 & Astringents and fevers \\
\hline $\begin{array}{l}\text { Fumaria indica (Hausskn.) } \\
\text { Pugsley }\end{array}$ & Papra & Herb & Persistent & $S, L$ & 180 & Cooling agent, fever and jaundice \\
\hline Geranium wallichianum D.Don. & Srajarrai & Herb & Persistent & $\mathrm{R}$ & 180 & Rheumatism \\
\hline Hedera nepalensis K. Koch. & Palul zelai & Climber & Increased & $\mathrm{L}$ & 70 & Cancer, jaundice and low bp \\
\hline Hyoscyamus niger $\mathrm{L}$. & Ajwain khuransani & Herb & Increased & $\mathrm{Fr}$ & 150 & $\begin{array}{l}\text { Sedative, Sleeplessness and } \\
\text { depression }\end{array}$ \\
\hline Juglans regia $\mathrm{L}$. & Ghoz & Tree & Increased & $\mathrm{L}, \mathrm{Fr}, \mathrm{W}$ & 550 & $\begin{array}{l}\text { Anti-helminthic, astringent, } \\
\text { antidiarrheal and hypoglycemic }\end{array}$ \\
\hline Justicia adhatoda L. & Bekan & Herb & Persistent & $\mathrm{L}$ & 120 & Rheumatism \\
\hline Morchella esculenta L. & Guji/Khosai & Fungi & Increased & WP & 35,000 & Aphrodisiac and tonic \\
\hline Morchella conia Pers. & Guji/Khosai & Fungi & Persistent & WP & 33000 & $\begin{array}{l}\text { Emollient, purgative, body tonic, } \\
\text { laxative }\end{array}$ \\
\hline Mentha longilfolia (L.) L. & Velanay & Herb & Increased & YS & 65 & Rheumatism \\
\hline Mentha spicata L. & Pudina & Herb & Persistent & & 70 & Rheumatism \\
\hline Myrtus communis L. & Manro & Shrub & Increased & $\mathrm{L}$ & 250 & Dysentery \\
\hline Malva sylvestris L. & Sonchal & Herb & Persistent & $\mathrm{L}$ & 80 & Digestion \\
\hline Malva neglecta Wall. & Panerak & Herb & Persistent & YS & 70 & Digestion \\
\hline Morus alba L. & Spin tout & Tree & Persistent & $\mathrm{Fr}$ & 90 & Astringent and tonic \\
\hline Morus nigra L. & Tour tout & Tree & Persistent & $\mathrm{Fr}$ & 90 & Aphrodisiac and tonic \\
\hline Olea ferruginea Wall. ex Aitch. & Khuna & Tree & Increased & $\mathrm{Ba}, \mathrm{L}, \mathrm{Tr}$ & 250 & Throat ache and skeleton disorders \\
\hline Onosma echioides $\mathrm{L}$. & Ratanjote/Gawzaban & Herb & Increased & $\mathrm{Fr}$ & 250 & Coetaneous eruption \\
\hline $\begin{array}{l}\text { Persicaria amplexicaulis (D. } \\
\text { Don) Ronse Decr. }\end{array}$ & Tarwa panrna & Herb & Increased & $\mathrm{Rh}$ & 400 & Rheumatism \\
\hline Prunus domestica L. & Alocha/Alobukhara & Shrub & Increased & $\mathrm{Fr}$ & 350 & Stomachic \\
\hline Paeonia emodi Wall. & Mamekh & Herb & Increased & $\mathrm{Rh}$ & 180 & Rheumatism \\
\hline $\begin{array}{l}\text { Sinopodophyllum hexandrum } \\
\text { (Royle) T.S. Ying }\end{array}$ & Bankakrri & Herb & Increased & $\mathrm{Rh}$ & 200 & Purgative/cancer \\
\hline $\begin{array}{l}\text { Pistacia integerrima St. ex } \\
\text { Brand. }\end{array}$ & Shnai & Tree & Increased & Pod & 500 & Cough \\
\hline
\end{tabular}


Ethnobotany Research and Applications

\begin{tabular}{|c|c|c|c|c|c|c|}
\hline Pinus roxburghii Sargent. & Nakhtar & Tree & Increased & Gu & 160 & Urticaria and tonic \\
\hline $\begin{array}{l}\text { Polygonatum multiflorum (L.) } \\
\text { All. }\end{array}$ & Nor-e-alam & Herb & Persistent & $\mathrm{Rh}$ & 250 & Placenta removal \\
\hline $\begin{array}{l}\text { Polygonatum verticillatum (L.) } \\
\text { All. }\end{array}$ & Nor-e-alam & Herb & Persistent & $\mathrm{Rh}$ & 250 & Placenta removal \\
\hline Punica granatum $\mathrm{L}$. & Nangoray/Anar & Shrub & Increased & $\mathrm{Fr}$ & 180 & $\begin{array}{l}\text { Anthelmintic/control urine in } \\
\text { children }\end{array}$ \\
\hline Portulaca oleracea L. & Warkharay/Culpa & Herb & Persistent & $\mathrm{S}, \mathrm{L}$ & 200 & Fever and digestion \\
\hline Rheum emodi Wall. & Rewand chini & Herb & Increased & $\mathrm{R}$ & 40 & Jaundice and liver diseases \\
\hline Rosa moschata Benth. & Gulab & Shrub & Persistent & $\mathrm{Fl}$ & 45 & Golkand \\
\hline Rheum webbianum Royle & Chotial/Rewand & Herb & Increased & $\mathrm{Rh}, \mathrm{L}$ & 45 & Liver diseases and jaundice \\
\hline Solanum nigrum L. & Kamachu & Herb & Increased & $\mathrm{Fr}$ & 120 & Tonic and kidney stone \\
\hline Solanum surattense Burm. f. & Marrorang & Herb & Increased & $\mathrm{Fr}$ & 120 & Demulcent \\
\hline $\begin{array}{l}\text { Salvia moorcroftiana Wall. ex } \\
\text { Benth. }\end{array}$ & Kharghwug & Herb & Increased & $\mathrm{S}, \mathrm{L}$ & 150 & Diarrhea and hemorrhoids \\
\hline Thymus linearis Benth. & Ghra sperkai & Herb & Increased & WP & 45 & Expectorant \\
\hline Taxus wallichiana Zucc. & Banya & Tree & Increased & $\mathrm{L}$ & 180 & Anthelmintic \\
\hline Valariana jatamansi Jones & Mashkebala & Herb & Increased & $\mathrm{Rh}$ & 600 & Nervous sedative \\
\hline Vitex negundo L. & Marwandai & Shrub & Persistent & $\mathrm{S}$ & 90 & Headache and febrifuge \\
\hline Viola canescens Wall. & Banafsha & Herb & Increased & $\mathrm{Fl}$ & 1000 & Diaphoretic and diuretic \\
\hline $\begin{array}{l}\text { Withania coagulans (Stocks) } \\
\text { Dunal }\end{array}$ & Kotilal & Shrub & Increased & $\mathrm{S}$ & 200 & Anodyne \\
\hline Withania somnifera (L.) Dunal & Kotilal & Herb & Increased & $S$ & 200 & Anodyne \\
\hline Ziziphus jujuba Mill. & Markhanai & Tree & Increased & $\mathrm{Fr}$ & 45 & Antidiabetic \\
\hline
\end{tabular}

Where: Ba, bark; C, corm; Fr, fruit; Fl, flower; Gu, gum; L, leaves; P, pod; R, root; Rh, rhizome; S, stem; WP, whole plant; YP, young plant; YS, young shoot 


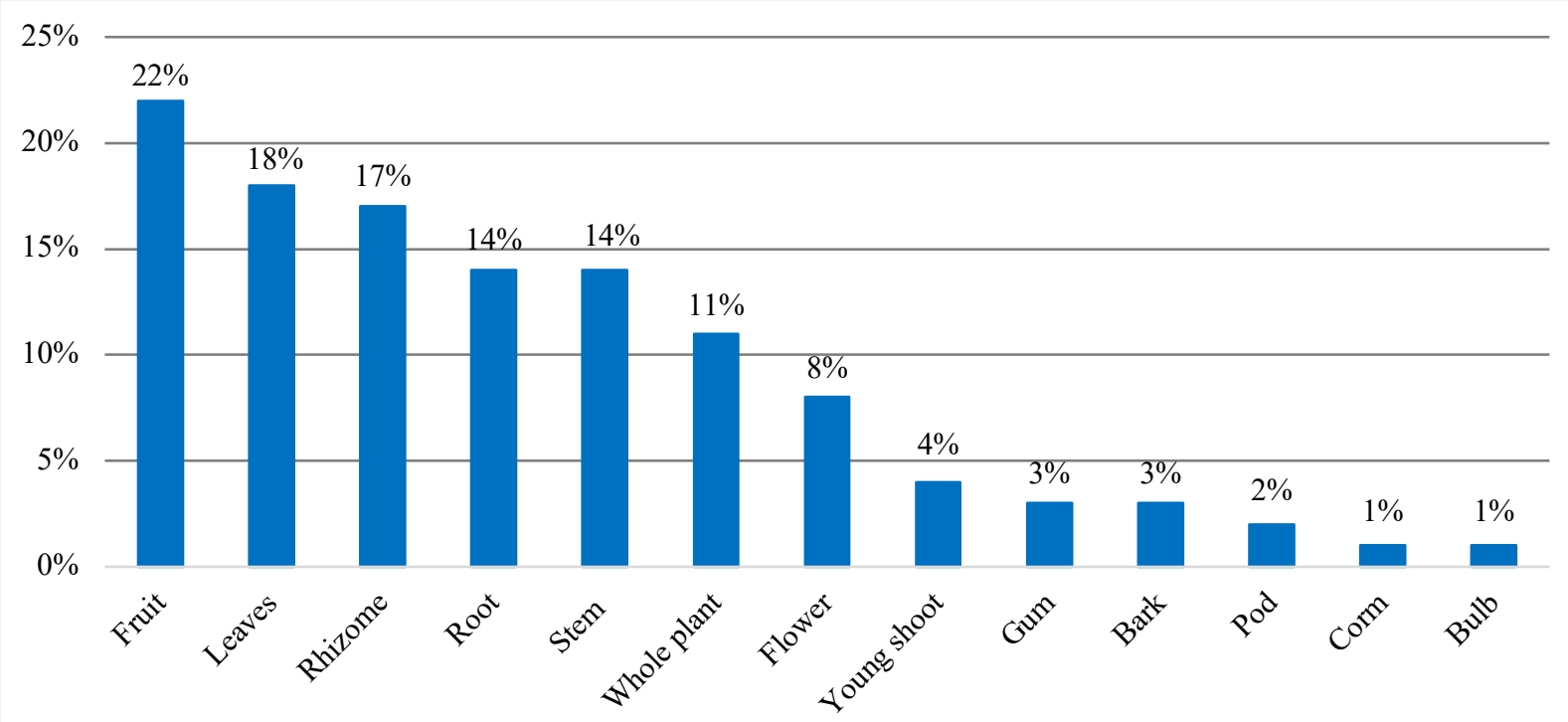

Figure 3. Percentage of different plant parts utilized by Hakims in various formulations.

\section{Specific MAPs which can contribute to poverty reduction}

Poverty alleviation efforts by government and private sectors in Swat have brought about significant improvement in the economy. However, poverty is still persistent and is higher in the rural areas where major portion of the population (about 70\%) depended on agriculture and farming. It was observed that significant number of the sample population was involved in collection and trade of MAPs from the wild (Table 3). The results showed that highest price was paid for Morchella esculenta, Viola canescens, Aconitum heterophyllum, Dioscorea deltoidea and Persicaria amplexicaulis which were collected in large quantities. The prices of each species varied from year to year and also depended on demand and supply. A brief set of information for 58 medicinal plants is given in Table 3 , for which market demand is increased/decreased or remained persistent.

\section{Price variations in the market of maps in trade chain from collectors to the end users}

The study reported that price of MAPs from collection to end users (traders, hakims and pharmaceutical companies) gradually increased at each step of the trade (Table 1,2,3). The price fluctuation was due to considerable loss of weight during drying, processing and packing. The low price at collector's level was influenced by improper collection and processing modes and having no knowledge of the value of the MAPs in the trade markets.

\section{Medicinal plants of swat exported to different countries}

Swat not only plays an important role in the supply and trade of medicinal herbs inside the country but is also a very important source for the export of medicinal herbs to different countries, however; it is a matter of concern to refer to it as non-documented economy of the country. The export of MAPs to different countries was observed to be in the hands of few individuals and exporters which hesitate to tell the truth about foreign trade routes and real price at international markets and their income. The market survey reported few species currently exported to different countries from District Swat directly or through national markets of the country including Peshawar, Islamabad, Lahore, and Karachi (Table 4).

\section{Discussion}

Swat being host to many important medicinal and aromatic plants, has a strong history of indigenous knowledge systems related to plants due to its centuries old traditions and culture. These medicinal herbs play an important role in the economic activity of the hillside dwellers in the valley and are the main source for traditional system of medicines. The results showed that the MAPs gatherers were mostly illiterate, and the collectors harvested medicinal plants unsustainably and improperly. The dealers of medicinal plants were also often not aware of the fact that collectors were unskilled and untrained and had no knowledge about the proper collection and harvesting practice of medicinal plants. As a result, about half of the collected material of medicinal herbs is wasted (Sher et al. 2015 and 2017). Poverty is the real and major cause, especially in the mountainous regions, that forces the people to harvest medicinal plants and to sell them at a very low price to middlemen to subsidize their low income and fulfil their basic needs of rural life (Nwafor et al. 2021). Most medicinal plants are used only locally, with a lesser number entering national or regional trade, and fewer still reaching international markets. However, in the last few 
decades, the trade of MAPs is receiving attention at national and international levels and medicinal plant trade plays a major role in global economy (Astutik et al. 2019).

Table 4. Important medicinal plants of district Swat exported to different countries

\begin{tabular}{|l|l|l|l|}
\hline Botanical names & Local names & $\begin{array}{l}\text { Part } \\
\text { used }\end{array}$ & Country of export \\
\hline Acacia modesta Wall. & Palosa & $\mathrm{Gu}$ & France, Dubai, India \\
\hline Aconitum heterophyllum Wall. & Zaharmora & $\mathrm{Rh}$ & Dubai, Germany, Saudi Arabia \\
\hline Aconitum laeve Royle. & Zaharmora & $\mathrm{Rh}$ & Dubai, Germany, Saudi Arabia \\
\hline Asparagus adscendens Roxb. & Muslisufaid & $\mathrm{Rh}$ & India, Middle East \\
\hline Colchicum luteum Baker. & $\begin{array}{l}\text { Suranjan-e- } \\
\text { talkh }\end{array}$ & $\mathrm{Rh}$ & $\begin{array}{l}\text { India, Italy, Germany, Saudi Arabia, Hong } \\
\text { Kong }\end{array}$ \\
\hline Dioscorea deltoidea Wall. & Kaneez & $\mathrm{Rh}$ & France, Dubai, Germany, \\
\hline Juglans regia L. & Ghoz & $\mathrm{Fr}$, Ba & India, Middle East \\
\hline Morchella esculenta L. & Gochi & $\mathrm{Fr}$ & France, Germany, Saudi Arabia, America, \\
\hline Paeonia emodi Wall. & Mamekh & $\mathrm{Rh}$ & Dubai, India, Germany, Saudi Arabia \\
\hline $\begin{array}{l}\text { Persicaria amplexicaulis (D. Don) } \\
\text { Ronse Decr. }\end{array}$ & Tarwa Panra & $\mathrm{Rh}$ & India, Japan, Germany, Hong Kong \\
\hline $\begin{array}{l}\text { Sinopodophyllum hexandrum (Royle) } \\
\text { T.S. Ying }\end{array}$ & Kakora & $\mathrm{Rh}$ & Dubai, Saudi Arab, Germany \\
\hline Taxus wallichiana Zucc. & Banrya & $\mathrm{L}$ & France, Germany, USA \\
\hline Viola canescens Wall. & Banafsha & $\mathrm{Fl}$ & $\begin{array}{l}\text { France, Dubai, Germany, India, Saudi } \\
\text { Arabia, Singapore }\end{array}$ \\
\hline Valariana jatamansi Jones & Mushkibala & $\mathrm{Rh}$ & France, Dubai, Saudi Arabia, Singapore \\
\hline
\end{tabular}

Where. Fr, fruit; Fl, flower; Gu, gum; L, leaves; Rh, rhizome; Ba, Bar

The study reported that people mostly rely on plants for medicines and use plants as a source of their income. With the advancement of pharmaceutical research, there is increasing exploitation of such resources and traditional medicines are getting rare (Sher et al. 2017; Salim et al. 2019). We found 160 species in the area that were used for the treatment of various diseases both in human and livestock disorders and also for earning income. However, their intensive utilization and over-harvesting have resulted increasing pressure on many plants. This population decrease of many economically and medicinally valuable plants have led to their collection more than one time a year (Hussain et al, 2007). The recent study showed that it is a matter of concern that most of the endemic plant species will continue to be collected in the future due to increased MAPs trade. Due to decreasing plant populations collectors now must use more effort, and to walk longer distances, to collect the same materials of targeted plants when compared to twenty years ago (Ghimire et al. 2016). There is an urgent need to find new strategies to remediate potential threats to medicinal herbs in. Rehabilitation and recovery practices are needed to rest plant diversity in general, and medicinal plants wealth. Natural vegetation of the study area is under heavy pressure because of overgrazing, illicit cutting, unabated urbanization and unauthorized collection of medicinal plants and conversion of forest land into agricultural land. There is a need to conserve these resources for the future, as they are refuge for valuable and endangered animals and birds and for our own survival (Sher et al. 2012; Hussain et al. 2018).

Market information in the present study revealed that Swat is the collection and trading centre for many medicinal and aromatic herbs. It has a well-established market which exports a large number of valuable plants to various national and international trading centres of herbal markets. It has previously reported that majority of marketable medicinal plants were collected from Northern areas of Pakistan including Swat (Sher et al. 2014). Our study revealed that trade of medicinal plants is complex and heterogeneous involving many players, and it was observed that the prices of targeted plants increased 3 to 5 times from collectors to local dealers. Collectors were selling the plants material to the wholesalers directly according to the demand of the species, or to middlemen then to retailers or pharmaceutical companies or exporters. The collectors earned a minimum in this chain, while the middlemen and wholesalers receive more. The price of medicinal and aromatic plants from collectors to the end users (traders, hakims and pharmaceutical companies) gradually increased at each step of the trade. The main reason of low price at collectors' level was due to improper collection and processing methods, and no knowledge of the real value of the MAPs in the markets. Sher et al. (2014) have also reported that middlemen and wholesalers earned high profits, and when these plants reached the international market, their prices highly increased. 
The study revealed that in most parts of the area especially at high elevation and remote areas, the main collectors were children, followed by women, men, and visiting collectors/outsiders. This agrees with the studies of Kunwar et al. (2018) who reported that males often collect medicinal plants from distant areas, whereas women collectors usually prefer accessible ranges. Ali et al. (2012) previously reported involvement of children (75\%), women (21\%), and older people (4\%) in collection activities of medicinal plants in Swat, Pakistan. They also recorded dominancy of collectors over dealers in trading activities. However, in contrary, it has been reported by Ahmad et al. (2014) that men in Philippines and Pakistan within the community are mainly responsible for medicinal plant collection while women play their role in treatment of ailments at household level.

\section{Conclusions}

The conducted research work revealed the current status of medicinal and aromatic plant in the study area, and it is in these localities that a wealth of different medicinal plant species is often found. It was recorded that almost all MAPs are neglected, over utilized, not properly documented and cultivation is almost non-existent, which has led to a diminution of many plant species and even disappearance of some of them. It was also concluded that its gathering and processing by rural women and their immediate family members has been identified as a possible source of human and livestock health maintenance, also as a source of cash income. The crude and sometimes untimely gathering of medicinal plants does little to enhance their survival or effective application for treatment of respective ailments. Biotic pressure from population growth and man's resulting domestic activities are placing some species under threat. The present study thus recommends initiating conservation measures with involvement of local communities to rehabilitate the exhausted plant resources. Further, training for collectors may be the best approach for sustainable management of MAPs and information on prices of marketable species.

\section{Declarations}

List of abbreviations: MAPs: Medicinal and aromatic plants; APG: Angiosperm Phylogeny Group Classification: APG-IV. Nomenclature: WFO: World Flora Online and POWO: Plants of the World Online

Approval and consent to participate: Prior verbal consent was obtained from all informants.

Competing interests: The authors declared no conflicting interests.

Funding: No funding was available for the present study.

Author's contributions: HS designed and conducted the study, including the initial data analyses. AA and ZU conducted additional data analyses and worked with HS on preparation of this paper. All authors have read and approved this version.

\section{Acknowledgements}

We greatly thank the local communities for sharing their valuable knowledge on wild medicinal and aromatic plants. Thanks are also due to the Forest Department, KP, Pakistan for secondary data of the study area.

\section{Literature cited}

Ahmad M, Sultana S, Fazl-i-Hadi S, Ben Hadda T, Rashid S, Zafar M, Khan MA, Khan, MPZ, Yaseen G. 2014. An Ethnobotanical study of Medicinal Plants in high mountainous region of Chail valley (District Swat-Pakistan). Journal of Ethnobiology and Ethnomedicine 10(1):1-18.

Ali H, Ahmad H, Marwat KB, Yousaf M, Gul B, Khan I. 2012. Trade potential and conservation issues of medicinal plants in district Swat, Pakistan. Pakistan Journal of Botany 44(6):1905-1912.

Ali SI. 2008. Significance of flora with special reference to Pakistan. Pakistan Journal of Botany. 40(3):967-71.

Amjad MS, Zahoor U, Bussmann RW, Altaf M, Gardazi SMH, Abbasi AM. 2020. Ethnobotanical survey of the medicinal flora of Harighal, Azad Jammu \& Kashmir, Pakistan. Journal of Ethnobiology and Ethnomedicine 16(1):1-28.

Astutik S, Pretzsch J, Ndzifon Kimengsi J. 2019. Asian medicinal plants' production and utilization potentials: A review. Sustainability 11(19):5483.

Aziz, MA, Adnan M, Khan AH, Rehman AU, Jan R, Khan J. 2016. Ethno-medicinal survey of important plants practiced by indigenous community at Ladha subdivision, South Waziristan agency, Pakistan. Journal of Ethnobiology and Ethnomedicine 12(1):1-14.

El-Assri EM, El Barnossi A, Chebaibi M, Hmamou A, El Asmi H, Bouia A, Eloutassi N. 2021. Ethnobotanical survey of medicinal and aromatic plants in Taounate, Pre-Rif of Morocco. Ethnobotany Research and Applications 22:1-23.

Ghimire SK, Awasthi B, Rana S, Rana HK, Bhattarai R, Pyakurel D. 2016. Export of medicinal and aromatic plant materials from Nepal. Botanica Orientalis-Journal of Plant Science 10:24-32. 
Government of Pakistan. 2017. Pakistan Bureau of Statistics, population census of district Swat, https://www.pbs.gov.pk/node/3391/?name=022

Hamilton AC. 2004. Medicinal plants conservation and livelihoods. Biodiversity and Conservation. 13:1477-1517.

Hussain F, Shah M, Sher H. 2007. Traditional resource evaluation of some plants of Mastuj, district Chitral, Pakistan. Pakistan Journal of Botany. 39(2):339-354.

Hussain W, Badshah L, Ullah M, Ali M, Ali A, Hussain F. 2018. Quantitative study of medicinal plants used by the communities residing in Koh-e-Safaid Range, northern Pakistani-Afghan borders. Journal of Ethnobiology and Ethnomedicine 14(1):1-18.

Islam M, Ahmad I, Akhtar N, Alam J, Razzaq A, Mohammad K, Mahmood T, Khan FU, Khan WM, Ahmad I, Ullah I, Shafaqat N, Qamar S. 2021. Medicinal plants resources of Western Himalayan Palas Valley, Indus Kohistan, Pakistan: Their uses and degrees of risk of extinction. Saudi Journal of Biological Sciences 28(5):3076-3093.

Ji Y, Fang Q, Liu S, Zhang B, Long C. 2020). Herbal medicinal markets in China: an ethnobotanical survey. Medicinal Plants: Biodiversity, Sustainable Utilization and Conservation, 415-429.

Khan MA, Khan MA, Hussain M, \& Ghulam GM. 2010. An ethnobotanical inventory of himalayan region poonch valley azad kashmir (Pakistan). Ethnobotany Research and Applications 8:107-123.

Khan J, Ghaffar A, \& Khan SA. 2018. The changing epidemiological pattern of Dengue in Swat, Khyber Pakhtunkhwa. PloS one, 13(4): e0195706.

Kunwar RM, Fadiman M, Cameron M, Bussmann RW, Thapa-Magar KB, Rimal B, Sapkota P. 2018. Cross-cultural comparison of plant use knowledge in Baitadi and Darchula districts, Nepal Himalaya. Journal of Ethnobiology and Ethnomedicine 14(1):1-17.

Larsen HO, Smith PD, \& Olsen CS. 2005. Nepal's conservation policy options for commercial medicinal plant harvesting: stakeholder views. Oryx, 39(4): 435-441.

Nasir E, Ali SI. 1971-1989. Flora of West Pakistan. No. 1-191. Department of Botany. University of Karachi, Karachi. Nwafor I, Nwafor C, Manduna I. 2021. Constraints to Cultivation of medicinal plants by smallholder farmers in South Africa. Horticulturae 7(12):531.

Rasul G, Choudhary D, Pandit BH, Kollmair M. 2012. Poverty and livelihood impacts of a medicinal and aromatic plants project in India and Nepal: an assessment. Mountain Research and Development 32(2):137-148.

Salim MA, Ranjitkar S, Hart R, Khan T, Ali S, Kiran C, Parveen A, Batool Z, Bano S, Xu J. 2019. Regional trade of medicinal plants has facilitated the retention of traditional knowledge: case study in Gilgit-Baltistan Pakistan. Journal of Ethnobiology and Ethnomedicine 15(1):1-33.

Shakya AK. 2016. Medicinal plants: Future source of new drugs. International Journal of Herbal Medicine 4(4):5964.

Sher H, Aldosari A, Ali A, de Boer HJ. 2014. Economic benefits of high value medicinal plants to Pakistani communities: an analysis of current practice and potential. Journal of Ethnobiology and Ethnomedicine 10:1-16.

Sher H, Aldosari A, Ali A, de Boer HJ. 2015. Indigenous knowledge of folk medicines among tribal minorities in Khyber Pakhtunkhwa, northwestern Pakistan. Journal of Ethnopharmacology 166:157-167.

Sher $\mathrm{H}$, Ali H, \& Rehman S. 2012. Identification and conservation of important plant areas (IPAS) for the distribution of medicinal, aromatic and economic plants in the Hindukush-Himalaya mountain range. Pakistan Journal of Botany 44(SI):187-194.

Sher H, Barkworth ME, \& de Boer HJ. 2017. Medicinal and aromatic plant cultivation in the Swat valley, northwestern Pakistan, for economic development and biodiversity conservation. Genetic Resources and Crop Evolution 64(2):237-245.

Sher H, Bussmann RW, Hart R, de Boer HJ. 2016. Traditional use of medicinal plants among Kalasha, Ismaeli and Sunni groups in Chitral District, Khyber Pakhtunkhwa province, Pakistan. Journal of Ethnopharmacology:188:57-69. Sher H, Hussain F, Sher H. 2010. Ex-situ management study of some high value medicinal plant species in Swat, Pakistan. Ethnobotany Research and Applications 8:017-024.

Uzun SP, Koca C. 2020. Ethnobotanical survey of medicinal plants traded in herbal markets of Kahramanmaraş. Plant Diversity 42(6):443-454.

Van Wyk AS, Prinsloo G. 2018. Medicinal plant harvesting, sustainability and cultivation in South Africa. Biological Conservation 227:335-342. 
ANNNEX - QUESTIONNAIRES

\section{Questionnaire No. 1: ETHNOGRAPHIC PROFILE OF INFORMANTS AND AREA} Name:

Locality:

Age:

Marital Status: single/Married

Gender: Male/ Female

Education: Illiterate/ Primary/Middle/High/Higher Secondary/bachelors/Masters

Occupation: Govt. Job/Private Job/Agriculture/Business/Others

Cast:

Tribe:

subtribe:

Household Composition

$\begin{array}{ll}\text { a. } & \text { Married Male } \\ \text { b. } & \text { Married Female } \\ \text { c. } & \text { Unmarried Boys: } \\ \text { d. } & \text { Unmarried Girls: } \\ \text { e. } & \text { Total: }\end{array}$

Source of income:

1. What is the total population of your village?

2. How many houses are there in your village?

3. How many family members lives per house?

4. What is per house per month income?

5. What is the main source of income?

6. How many different ethnic groups live in your area?

7. What type of trees/ vegetation is there?

8. How you maintain its protection?

9. Are the people of area converting forest area into cultivated fields?

10. Change in population of human beings for the last 10 years? (Increased/Decreased/persistent)

11. What are the causes of change in population?

12. What are the responsibilities of women outside the house in your area?

13. At what age Male and Females get married?

14. What type of human diseases are common in your area?

15. What do people do if they are sick?

16. To whom do they consult?

17. Are there any local healers and ritual specialists etc?

18. What are the major commercial cultivation and crops of your area?

19. What types of animals are mostly reared in your area?

20. Why are such type of veterinary diseases common in your area?

21. What do people do if animals got sick?

22. Is there any environmental difference in the last $10-20$ years in your area?

23. If yes, what are the differences?

24. Is the usage of plants for various purposes by locals are increasing/decreasing/remains the same?

25. If deceases then why?

26. What type of people have more ethnobotanical knowledge?

27. Why they have more ethnobotanical knowledge?

28. From where they get such knowledge? 


\section{Questionnaire No. 2: MEDICINAL PLANTS OF THE AREA}

1. What are the most recent diseases, your family members, suffered from?

2. What kind of treatment you get? Allopathic/ Herbal/ Religious/ Homeopathic

3. Have you got any herbal medicine, why? Easily available / Most effective/ Free of cost /All of them/

Other

4. How many medicinal Plants you know? Table

a. Specie Name?

b. Price/KG?

c. Growing season?

d. Use value? Abundant/common/scarce/rare etc?

e. Cultivated/wild/weed?

f. Found in Forests/Range Land/Pastures/waste land/gardens/others?

g. Who collect Men/Women/Children/Herbalist/Others?

5. The uses of medicinal plants are increasing/deceasing/remains the same?

6. If deceasing then why?

7. How you got the knowledge about the use of medicinal plants?

8. Which medicinal plants are abundantly used in this area? Table

a. Specie name?

b. Reason of usage?

c. Used for the type of disease?

d. Result of usage? $20 \% 40 \%, 60 \%, 80 \%, 100 \%$

e. Personally used it in Men/Women/Children?

f. Side effect of this medicinal plant?

9. Are medicinal plants used extensively/Commonly/ scarcely/rarely in area?

10. Which plants are used extensively?

11. Why are these plants used extensively?

12. Is the population of these plants deceasing? Yes/No

13. If yes, why these are decreasing?

14. How can we conserve them?

15. Name and part of the plant used for medicines. Table

a. Name of the specie

b. Disease

c. Tree/shrub/Herbs?

d. Part used: Leaves/Fruits/Seeds/Flower/roots/whole plant?

e. Recipe

16. What are the most recent diseases, your family members, suffered from?

17. What kind of treatment you get? Allopathic/ Herbal/ Religious/ Homeopathic

18. Have you got any herbal medicine, why? Easily available / Most effective/ Free of cost /All of them/

Other

19. How many medicinal Plants you know? Table

a. Specie Name?

b. Price/KG?

c. Growing season?

d. Use value? Abundant/common/scarce/rare etc.?

e. Cultivated/wild/weed?

f. Found in Forests/Range Land/Pastures/waste land/gardens/others?

g. Who collect Men/Women/Children/Herbalist/Others?

20. The uses of medicinal plants are increasing/deceasing/remains the same?

21. If deceasing then why?

22. How you got the knowledge about the use of medicinal plants?

23. Which medicinal plants are abundantly used in this area? Table 
Questionnaire No. 3: SPECIFIC INFORMATION OBTAINED FROM HAKIMS

1. For how long, are you doing this practice?

2. How you gained this knowledge?

3. Any historical practice in your family before you?

4. Do you collect medicinal plants yourself?

5. Who bring plants and herbal material to you?

6. Do you also purchase plants from the herbal market?

7. Are all the plants locally collected or also come from other region? From India?

8. Do you think your purchase rates of medicinal plants from local collectors are reasonable?

9. From which areas different collected plants come to you?

10. Which type of people mostly sell plants to you?

11. From which areas most of your patients come?

12. Do you think the collected plants are properly collected?

13. Are the collectors know the best time of collection?

14. Do collectors, collect only the useful part of the plant?

\section{Questionnaire No. 4: SPECIFIC INFORMATION OBTAINED FROM TRADERS}

1. For how many years you are involved in the business?

2. How many MAPs species you have in your store?

3. From where they come?

4. From whom you purchase them?

5. How much quantity of MAPs you purchase each year?

6. How much quantity you sell each year?

7. How much quantity do you export to foreign countries?

8. What are the rates per/ $\mathrm{Kg}$ of various plants?

9. How many species are exported abroad?

10. Are you satisfied with quality of collection?

11. Do you think some species are collected in huge quantity?

12. Are these maps come from wild or cultivation?

13. Who are the buyers at your shop? 